\title{
A BOUNDARY VALUE PROBLEM FOR SYMMETRIC ELLIPTIC SYSTEMS OF FIRST ORDER SEMILINEAR PARTIAL DIFFERENTIAL EQUATIONS IN OPEN SETS OF CLASS $C^{1}$
}

\author{
R. SELVAGGI AND I. SISTO ${ }^{1}$
}

\begin{abstract}
Si dimostrano un teorema di unicità ed un teorema di esistenza per sistemi ellittici simmetrici a coefficienti costanti di equazioni alle derivate parziali del primo ordine semilineari in aperti di classe $C^{1}$ e con dati al contorno in $L^{p}$.
\end{abstract}

Introduction. An existence and uniqueness theorem was established in [3] for a bcundary value problem related to an elliptic system of first order semilinear partial differential equations. The problem was considered in a bounded open subset $\Omega$ of $\mathbf{R}^{m}(m \geq 3)$ of class $C^{2}$ and boundary data of class $C^{1}$.

The study of a boundary value problem for first order linear elliptic systems with constant coefficients in bounded open sets of class $C^{1}$ carried out by the present authors in [7], suggested the extension of the results obtained in [3] to the case in which $\Omega$ is of class $C^{1}$ and the boundary data are in $L^{p}$.

In this paper, we prove an existence and uniqueness theorem for that problem, making use of the integral representation used by A. Avantaggiati in [2] for the solutions of the linear system considered there.

1. Consider the following problem:

I. Let $\Omega$ be a bounded open subset of $\mathbf{R}^{m}(m \geq 3)$ of class $C^{1}$; let $f=\left(f_{r}\right)_{1 \leq r \leq 2 n}$ be a finite sequence of functions such that

(i) $f_{r}(X, u)$ is continuous in $u$ for almost every $X \in \Omega$ and is measurable in $X$ for any $u \in \mathbf{R}^{2 n}$;

(ii) if $2 \leq s<+\infty$ and $t=s m /(m-1), a b>0$, an $h \in] 1-\frac{1}{t}, 1\left[\right.$ and an $a_{r} \in L^{s}(\Omega)$ exist such that

$$
\left|f_{r}(X, u)\right| \leq a_{r}(x)+b|u|^{h}
$$

let $B=\left(b_{k q}\right)_{1 \leq k \leq n ; 1 \leq q \leq 2 n}$ be a matrix whose elements are of class $C^{0}(\partial \Omega)$ with rank equal to $n$ at any point of $\partial \Omega$ and let $b_{0}=\left(b_{k 0}\right)_{1 \leq k \leq n} \in\left(L^{s}(\partial \Omega)\right)^{n}$. Our problem is to determine a $2 n$-tuple $u=\left(u_{q}\right)_{1 \leq q \leq 2 n} \in\left(L^{t}(\Omega)\right)^{2} n$ with first order derivatives a.e. in $\Omega$, which satisfies an elliptic symmetric system of first order semilinear differential equations with real constant coefficients

$$
\sum_{q=1}^{2 n} \sum_{p=1}^{m} a_{r q}^{p} \frac{\partial u_{q}}{\partial x_{p}}(X)=f_{r}(X, u), \quad r=1, \ldots, 2 n,
$$

a.e. in $\Omega$, and is such that

Received by the editors December 30, 1982.

1980 Mathematics Subject Classification. Primary 35J65, 45P05.

${ }^{1}$ Work supported by G.N.A.F.A. (C.N.R.).

(C) 1984 American Mathematical Society $0002-9939 / 84 \$ 1.00+\$ .25$ per page 
(i $i_{1}$ ) for any $\left.\alpha \in\right] 0,1[$, a $\delta>0$ exists such that for any $q \in\{1, \ldots, 2 n\}$ the maximal nontangential function of $u_{q}$,

$$
u_{q}^{*}(P)=\sup \left\{\left|u_{q}(X)\right|: X \in C_{\alpha}(P) \cap B(P, \delta)\right\},
$$

belongs to $L^{s}(\partial \Omega)$ (here $C_{\alpha}(P)=\{X \in \Omega:(X-P) \cdot N(P)>\alpha|X-P|\}$ where $N(P)$ is the inner normal at $P$ to $\partial \Omega$ and $B(P, \delta)$ is the open ball centered at $P$ and with radius $\delta$ ),

$\left(\mathrm{i}_{2}\right)$ for almost every $P \in \partial \Omega$ and for any $q \in\{1, \ldots, 2 n\}$, the limit

$$
\lim _{X \rightarrow P ; X \in C_{\alpha}(P)} u_{q}(X)
$$

exists; if we denote this limit by $u_{q}(P)$ it satisfies the following conditions a.e. in $\Omega$ :

$$
\sum_{q=1}^{2 n} b_{k q}(P) u_{q}(P)=b_{k 0}(P), \quad k=1, \ldots, n .
$$

2. Let

$$
a(N(P))=\left(a_{r q}(N(P))\right)_{1 \leq r, q \leq 2 n}=\left(\sum_{p=1}^{m} a_{r q}^{p} N_{p}(P)\right)_{1 \leq r, q \leq 2 n}
$$

where $N(P)=\left(N_{1}(P), \ldots, N_{m}(P)\right)$. Denote by $B^{*}$ the transpose of $B$, by $I$ the unit matrix of order $2 n$ and by 0 the null matrix of order $n$. If

$$
D(P, \rho)=\operatorname{det}\left(\begin{array}{cc}
a(N(P))-\rho I & B^{*}(P) \\
B(P) & 0
\end{array}\right)
$$

the following theorem holds.

THEOREM 2.1. If for any $P \in \partial \Omega$ the equation $D(P, \rho)=0$ admits only positive (resp. negative) roots and if $u \in \mathbf{R}^{2 n} \mapsto f(X, u)$ is an increasing (resp. decreasing) monotone function for almost every $X \in \Omega,{ }^{2}$ then problem $\mathrm{I}$ has at most one solution.

Proof. If $u^{1}$ and $u^{2}$ are solutions of problem I, since we have, a.e. in $\Omega$,

$$
\begin{aligned}
\sum_{r, q=1}^{2 n} \sum_{p=1}^{m} a_{r q}^{p} \frac{\partial}{\partial x_{p}} & {\left[\left(u_{q}^{1}(X)-u_{q}^{2}(X)\right) \cdot\left(u_{r}^{1}(X)-u_{r}^{2}(X)\right)\right] } \\
& =2\left(f\left(X, u^{1}(X)\right)-f\left(X, u^{2}(X)\right)\right) \cdot\left(u^{1}(X)-u^{2}(X)\right)
\end{aligned}
$$

and since, due to (i) and (ii), $f\left(\cdot, u^{i}(\cdot)\right) \in L^{t^{1}}(\Omega)(i=1,2)$ with $1 / t^{1}+1 / t=1$, we have that the function

$$
\sum_{r, q=1}^{2 n} \sum_{p=1}^{m} a_{r q}^{p}\left(u_{q}^{1}(X)-u_{q}^{2}(X)\right) \cdot\left(u_{r}^{1}(X)-u_{r}^{2}(X)\right)
$$

belongs to $W^{1,1}(\Omega)$. Hence, by means of arguments similar to those used in [3, Theorem 2.1], this theorem follows.

\footnotetext{
${ }^{2}$ If $H$ is a Hilbert space, we say that $f: H \rightarrow H$ is an increasing (resp. decreasing) monotone function, if it satisfies the following condition: $\forall u, v \in H: u \neq v \Rightarrow(f(u)-f(v)) \cdot(u-v)>0$ (resp. $<0)$.
} 
3. Let $M=\left(M_{r q}\right)_{1 \leq r, q \leq 2 n}$ be the fundamental matrix of the system (1.2) defined by $\left(5.1^{\prime \prime}\right)$ in [2]. We note that the following properties were proved in [2]:

$\left(\alpha_{1}\right) M_{r s}(\lambda X)=\lambda^{1-m} M_{r s}(X), \lambda>0$,

$\left(\alpha_{2}\right) M_{r s}(-X)=-M_{r s}(X)$,

$\left(\alpha_{3}\right) M_{r s}$ is an analytic function in $\mathbf{R}^{m}-\{0\}$.

Let $f$ be the vector which appears in (1.2). The following proposition holds:

Proposition 3.1. If we set

$$
\begin{gathered}
F_{1} e(X)=\int_{\Omega} M(X-Y) f(Y, e(Y)) d Y, \\
F_{2} \psi(X)=\int_{\partial \Omega} M(Q-X) \psi(Q) d Q
\end{gathered}
$$

a.e. in $\Omega$, we have that

$\left(i_{3}\right) F_{1}$ and $F_{2}$ are continuous operators from $\left(L^{t}(\Omega)\right)^{2 n}$ into $\left(H^{1, s}(\Omega)\right)^{2 n}$ and from $\left(L^{s}(\partial \Omega)\right)^{2 n}$ into $\left(L^{t}(\Omega)\right)^{2 n,}{ }^{3}$ respectively,

(i $\left.i_{4}\right)$ a constant $c>0$ exists such that

$$
\left\|F_{1} e\right\|_{\left(H^{1, s}(\Omega)\right)^{2 n}} \leq c\left(\|a\|_{\left(L^{\cdot}(\Omega)\right)^{2 n}}+\|e\|_{\left(L^{t}(\Omega)\right)^{2 n}}^{h}\right)
$$

$$
\left\|F_{2} \psi\right\|_{\left(L^{t}(\Omega)\right)^{2 n}} \leq c\|\psi\|_{\left(L^{s}(\partial \Omega)\right)^{2 n}}
$$

with $a=\left(a_{r}\right)_{1 \leq r \leq 2 n}$.

Proof. The statement for $F_{2}$ is an obvious consequence of Proposition 2.v in [4] which holds also in the case in which $\Omega$ is of class $C^{1}$. Furthermore, by (i) and (ii) the map $e \mapsto f(\cdot, e(\cdot))$ is continuous from $\left(L^{t}(\Omega)\right)^{2 n}$ into $\left(L^{s}(\Omega)\right)^{2 n}$ and by 3.IV in [4] the operator $\alpha \mapsto \int_{\Omega} M_{r q}(X-Y) \alpha(Y) d Y$ is continuous from $L^{s}(\Omega)$ into $H^{1, s}(\Omega)$. These facts immediately imply inequality (3.3).

In the sequel we will assume that for the quadratic form

$$
\sum_{r, q=1}^{2 n} a_{r q}(N(P)) u_{r} u_{q}
$$

the following hypothesis is satisfied:

$\left(\mathrm{I}_{1}\right)$ Any root of the equation $\operatorname{det}(a(N(P))-\rho I)=0$ has a constant multiplicity with respect to $P \in \partial \Omega$.

Hence a finite covering $\left\{B_{1}, \ldots, B_{N}\right\}$ of $\partial \Omega$ exists made up of coordinate neighborhoods and for any $j \in\{1, \ldots, N\}$ a matrix $\left(d_{q r}^{j}\right)_{1 \leq q \leq 2 n, 1 \leq r \leq n}=d^{j}$ of functions of class $C^{0}\left(B_{j}\right)$ exists with rank $n$ at any point of $B_{j}$ and for any $B_{j} \cap B_{i} \neq \varnothing$ an orthogonal matrix $\left(\vartheta_{l k}^{j i}\right)_{1 \leq l, k \in n}$ of class $C^{0}\left(B_{j} \cap B_{i}\right)$ exists such that

$$
d_{q r}^{i}=\sum_{l=1}^{n} \vartheta_{l r}^{j i} \cdot d_{q l}^{j}, \quad \forall r \in\{1, \ldots, n\} \forall q \in\{1, \ldots, 2 n\}
$$

(see n.5 of $[7])$.

${ }^{3} H^{1, s}(\Omega)$ is the completion of $C^{1}(\bar{\Omega})$ with respect to the norm

$$
\|f\|_{H^{1, s}(\Omega)}=\|f\|_{L^{s}(\Omega)}+\sum_{|\alpha|=1}\left\|D^{\alpha} f\right\|_{L^{s}(\Omega)} .
$$


We set, for any $e \in\left(L^{t}(\Omega)\right)^{2 n}$ and any $\varphi \in L^{s}\left(\{\vartheta\}_{B}\right)^{4,5}$

$$
u(X)=\int_{\partial \Omega} M(Q-X)(d \cdot \varphi)(Q) d Q+\int_{\Omega} M(X-Y) f(Y, e(Y)) d Y \quad \text { a.e. in } \Omega \text {. }
$$

If we require the vector $u$ to satisfy the boundary conditions (1.3) and if we take into account (3.5) in [6] and $\left(\alpha_{1}\right),\left(\alpha_{2}\right),\left(\alpha_{3}\right)$, for almost every $P \in \partial \Omega$, we have

$$
B \cdot C \cdot(d \cdot \varphi)(P)+\int_{\partial \Omega}^{*} B(P) M(P-Q)(d \cdot \varphi)(Q) d Q=g(P)
$$

where

$$
\begin{gathered}
\int_{\partial \Omega}^{*} B(P) M(P-Q)(d \cdot \varphi)(Q) d Q=\lim _{\epsilon \rightarrow 0^{+}} \int_{\partial \Omega-I(P, \epsilon)} B(P) \cdot M(P-Q)(d \cdot \varphi)(Q) d Q \\
C(P)=-\int_{\pi_{p}}^{*} M(P-Q+N(P)) d Q=-\lim _{\epsilon \rightarrow \infty} \int_{\pi_{p}-C(P, \epsilon)} M(P-Q+N(P)) d Q \\
g(P)=b_{0}(P)-B(P) \int_{\Omega} M(P-Y) f(Y, e(Y)) d Y
\end{gathered}
$$

$I(P, \epsilon)$ is the portion of $\partial \Omega$ having as a projection on the tangent plane $\pi_{p}$ to $\partial \Omega$ at $P$, the ball with center $P$ and radius $\epsilon>0$ and $C(P, \epsilon)$ is the ball of $\pi_{p}$ with center $P$ and radius $\epsilon>0$.

REMARK 3.1. From Proposition 3.1 and trace theorems it follows that the function defined in (3.10) belongs to $\left(L^{s}(\partial \Omega)\right)^{n}$ and

$$
\|g\|_{\left(L^{s}(\partial \Omega)\right)^{n}} \leq c_{1}\left(\left\|b_{0}\right\|_{\left(L^{s}(\partial \Omega)\right)^{n}}+\|a\|_{\left(L^{s}(\Omega)\right)^{2 n}}+\|e\|_{\left(L^{t}(\Omega)\right)^{2 n}}^{h} .\right.
$$

In the sequel we assume the symbolic matrices

$$
\mathcal{M}^{j}(P, \tau)=B(P) \cdot \Psi(P, \tau) \cdot d^{j}(P) \quad \forall P \in B_{j} \forall \tau \in \mathbf{R}^{m-1} \text { with }|\tau|=1
$$

associated with the singular integral equations (3.7), where $\Psi(P, \tau)$ is the symbolic matrix of the system of singular integral operators on $\partial \Omega$ (see n.3 of [7]) given by ${ }^{6}$

${ }^{4} L^{s}\left(\{\vartheta\}_{B}\right)=\left\{\varphi=\left(\varphi^{j}\right)_{1 \leq j \leq N} \in \prod_{j=1}^{N}\left(L^{j}\left(B_{j}\right)\right)^{n}: \varphi^{j}=\vartheta^{j i} \varphi^{i}\right.$ a.e. in $\left.B_{i} \cap B_{j}\right\}$.

${ }^{5} d \cdot \varphi$ is the vector of $\left(L^{s}(\partial \Omega)\right)^{2 n}$ whose restriction to $B_{j}$ coincides with $d^{j} \cdot \varphi^{j}$.

${ }^{6} \mathrm{The}$ symbol of the following operator $A_{n s}$ on $\partial \Omega$

$$
A_{r s} f(P)=f(P) \int_{\pi_{p}}^{*} M_{r s}(P-Q+N(P)) d Q+\int_{\partial \Omega}^{*} M_{r s}(P-Q) f(Q) d Q
$$

is the function on the cotangent bundle of $\partial \Omega$ defined by

where

$$
\Psi_{r s}\left(P, \sum \xi_{i} d x_{i}\right)=a_{r s}(x(P))+\lim _{\epsilon \rightarrow 0^{+}} \int_{\epsilon<|\eta|<1 / \epsilon} e^{i \xi \cdot \eta} h(x(P), \eta) d \eta
$$

$$
\begin{aligned}
a_{r s}(x)=\sqrt{1+|\nabla \varphi(x)|^{2}}\left(\int_{|z|<1} M_{r s}(z,\right. & +\nabla \varphi(x) \cdot z) d z \\
& \left.+\int_{|z|>1}\left[M_{r s}(z, 1+\nabla \varphi(x) \cdot z)-M_{r s}(z, \nabla \varphi(x) \cdot z)\right] d z\right), \\
h(x, z)=\sqrt{1+|\nabla \varphi(x)|^{2}} & M_{r s}(z, \nabla \varphi(x) \cdot z)
\end{aligned}
$$

and $P$ is in a coordinate neighborhood $V$ with coordinates $x$ such that with respect to this coordinate system

$$
V \cap \Omega=\left\{(x, t): x \in \mathbf{R}^{m-1}, t>\varphi(x)\right\} \cap V
$$

where $\varphi \in C_{0}^{1}\left(\mathbf{R}^{m-1}\right), \varphi(0)=\partial \varphi(0) / \partial x_{i}=0, i=1, \ldots, m-1$. 


$$
A: f \in\left(L^{s}(\partial \Omega)\right)^{2 n} \rightarrow C(P) \cdot f(P)+\int_{\partial \Omega}^{*} M(Q-P) f(Q) d Q
$$

satisfy the following assumption

$\left(\mathrm{I}_{2}\right)$ For any $P \in \partial \Omega, \tau \in \mathbf{R}^{m-1}$ with $|\tau|=1$ and $P \in B_{j}, \mathcal{M}^{j}(P, \tau) \neq 0$.

Furthermore, suppose that for any $P \in \partial \Omega$ the equation $D(P, \rho)=0$ admits only positive (resp. negative) roots and that this further assumption is satisfied:

$\left(\mathrm{I}_{3}\right)$ For almost every point $P \in \partial \Omega$ the quadratic form

$$
\sum_{j, l=1}^{n} \sum_{r, q=1}^{2 n} A_{r q}(N(P)) b_{j r}(P) b_{l q}(P) \lambda_{j} \lambda_{l}
$$

is positive (resp. negative) definite (where $\left(A_{r q}(N(P))\right)_{1 \leq r, q \leq 2 n}$ is the inverse matrix of $a(N(P)))$.

Under the above assumptions the function $S$ which to any $\varphi \in L^{s}\left(\{\vartheta\}_{B}\right)$ associates the element of $\left(L^{s}(\partial \Omega)\right)^{n}$ which appears on the left-hand side of $(3.7)$ is injective and the equation $S(\varphi)=g$ with $g$ given by (3.10) admits one and only one solution thanks to Remark (5.3) in [7]. Furthermore $S$ is surjective: actually, by an argument similar to that used in [2] and taking into account the results obtained in [7], we obtain that the theorem holds for $s=2$, since the transposed homogeneous system associated with $S \varphi=g$ has only the trivial solution. For $s \geq$ 2, if $g \in\left(L^{s}(\partial \Omega)\right)^{n}$ and $\varphi \in L^{2}\left(\{\vartheta\}_{B}\right)$ is the only solution of the equation $S \varphi=g$, by an argument similar to that used to show Theorem 5.1 in [7] it follows that $\varphi \in L^{s}\left(\{\vartheta\}_{B}\right)$. These facts imply that $S^{-1}$ is continuous and then an $H>0$ exists such that

$$
\|\varphi\|_{L^{s}\left(\{\vartheta\}_{B}\right)} \leq H\|g\|_{\left(L^{s}(\partial \Omega)\right)^{n}}
$$

Proposition 3.2. If we set

$$
T(e)=F_{1}(e)+F_{2}(d \cdot \varphi)
$$

where $\varphi$ is the unique solution of system (3.7), then

$\left(\mathrm{i}_{5}\right) T$ is a continuous operator from $\left(L^{t}(\Omega)\right)^{2 n}$ into itself,

(i6) a $c_{2}>0$ exists such that

$$
\|T(e)\|_{\left(L^{t}(\Omega)\right)^{2 n}} \leq c_{2}\left(\left\|b_{B}\right\|_{\left(L^{s}(\partial \Omega)\right)^{n}}+\|a\|_{\left(L^{s}(\Omega)\right)^{2 n}}+\|e\|_{\left(L^{t}(\Omega)\right)^{2 n}}^{h} .\right.
$$

Proof. Let $\left(e_{l}\right)_{l \in \mathbf{N}}$ be a sequence of elements of $\left(L^{t}(\Omega)\right)^{2 n}$ converging there to $e$. From Proposition 3.1 it follows that $\left(F_{1}\left(e_{l}\right)\right)_{l \in \mathbf{N}}$ converges to $F_{1}(e)$ in $\left(H^{1, s}(\Omega)\right)^{2 n}$. As a consequence, $\left(F_{1}\left(e_{l}\right)\right)_{l \in \mathbf{N}}$ converges to $F_{1}(e)$ in $\left(L^{t}(\Omega)\right)^{2 n}$ and

$$
\int_{\Omega} M(P-Y) f\left(Y, e_{l}(Y)\right) d Y
$$

converges to $\int_{\Omega} M(P-Y) f(Y, e(Y)) d Y$ in $\left(L^{s}(\partial \Omega)\right)^{2 n}$. The statement (i $\left.\mathrm{i}_{5}\right)$ follows from (3.10) and the continuity of $S^{-1}$ and $F_{2}$, while $\left(\mathrm{i}_{6}\right)$ is an obvious consequence of (3.3), (3.4), (3.11) and (3.12).

Choose $\rho>0$ such that ${ }^{7}$

$$
\|e\|_{\left(L^{t}(\Omega)\right)^{2 n}} \leq \rho \Rightarrow\|T(e)\|_{\left(L^{t}(\Omega)\right)^{2 n}} \leq \rho .
$$

\footnotetext{
${ }^{7}$ The existence of $\rho$ is ensured by (3.13) since $\left.h \in\right] 0,1[$.
} 
Proposition 3.3. With the same notations as in Proposition 3.2, if we set

$$
E=\left\{e \in\left(L^{t}(\Omega)\right)^{2 n}:\|e\|_{\left(L^{t}(\Omega)\right)^{2 n}} \leq \rho\right\},
$$

then

(i $) T(E) \subset E$,

(i 8$)$ The restriction of $T$ to $E$ has at least one fixed point.

Proof. ( $\left.i_{7}\right)$ is an immediate consequence of (3.14). As far as ( $\left.i_{8}\right)$ is concerned, since $E$ is closed and convex, it suffices, thanks to the Schauder fixed point theorem, to show that $T(E)$ is relatively compact. To this aim let $\left(e_{l}\right)_{l \in \mathbf{N}}$ be a sequence of elements of $E$. Since, by $(3.3),\left(F_{1}\left(e_{l}\right)\right)_{l \in \mathbf{N}}$ is bounded in $\left(H^{1, s}(\Omega)\right)^{2 n}$, it follows that $\left(F_{1}\left(e_{l}\right)\right)_{l \in \mathbf{N}}$ has a subsequence which converges in $\left(L^{t}(\Omega)\right)^{2 n}$ thanks to the Sobolev embedding theorems and also $\left(\int_{\Omega} M(P-Y) f\left(Y, e_{l}(Y)\right) d Y\right)_{l \in \mathbf{N}}$ has a subsequence which converges in $\left(L^{s}(\partial \Omega)\right)^{2 n}$ thanks to the well-known trace theorems. From (3.10), (3.13) and the continuity of $S^{-1}$ and $F_{2}$ it follows that $\left(T\left(e_{l}\right)\right)_{l \in \mathrm{N}}$ has a subsequence converging in $\left(L^{t}(\Omega)\right)^{2 n}$.

At last the following theorem holds:

TheOREM 3.1. Problem I admits at least one solution.

Proof. If $u$ is a fixed point of $T$ (see Proposition 3.3), then $u$ is a solution of Problem I.

\section{BIBLIOGRAPHY}

1. A. Ambrosetti and G. Prodi, Analisi non lineare, Ann. Scuola Norm. Sup. Pisa Cl. Sci. (4) (1973).

2. A. Avantaggiati, Problemi al contorno per $i$ sistemi ellittici simmetrici etc., Ann. Mat. Pura Appl. (4) 61 (1963), 193-258.

3. G. Caradonna, Un problema al contorno per $i$ sistemi ellittici di equazioni semi lineari alle derivate parziali del primo ordine, Note di Mat. 1 (1981), 187-202.

4. C. Miranda, Sulle proprietà di regolarità di certe trasformazioni integrali, Atti. Accad. Naz. Lincei Mem. Cl. Sci. Fis. Mat. Natur. Ser. I (8) 7 (1965), 303-336.

5. J. Necas, Les méthodes directes en théorie des équations elliptiques, Masson, Paris; Academia, Prague, 1967.

6. R. Selvaggi and I. Sisto, Regolarità di certe trasformazioni integrali relative ad aperti di classe $C^{1}$, Rend. Acad. Sci. Fis. Mat. Napoli (4) 45 (1978), 393-410.

7. $\ldots$, Problemi al contorno per $i$ sistemi ellittici simmetrici del primo ordine etc., Note di Mat. 7 (1981), 155-185.

Dipartimento di Matematica, Universita Degli Studi di Bari, Palazzo Ateneo, 70121 BARI, ITALY 